\title{
A 3D-Hole Closing Algorithm
}

\author{
Zouina Aktouf, Gilles Bertrand and Laurent Perroton \\ ESIEE Cité Descartes, B.P 9993162 Noisy-Le-Grand Cedex France \\ E-mail : \{aktoufz, bertrang, perrotol\}@esiee.fr
}

\begin{abstract}
Contrary to the 2D case, a 3D hole is not a subset of the 3D space. It is therefore not possible to use connected component search algorithms for detecting and suppressing 3D holes.

In this paper, we propose an algorithm for closing $3 \mathrm{D}$ holes. It is based on properties of the previously introduced notion of topological numbers. Our algorithm is linear in time and it allows to control the size of the holes which are closed. As far as we know, this is the first 3D-hole closing algorithm.
\end{abstract}

\section{Introduction}

In 3D image analysis, the reconstructed objects which result from the segmentation process have sometimes unwanted holes. These holes may be considered as an effect of noise. In many applications, the noisy holes can be characterized by their size: larger holes are actually features of the object, whereas a large amount of small holes are irrelevant.

In our approach, we consider the notion of hole from a topological point of view. It is important to make the difference between holes, cavities and concavities. Concavities are concave shapes of the contour of the object. Cavities are hollows in the object, or more formally, bounded connected components of the background. An object has a hole whenever it contains a closed path which cannot be transformed to a single point by a sequence of elementary deformations inside the object. For example, a ring has one hole and no cavity, whereas a hollow torus with one cavity has two holes. The closing of holes in $3 \mathrm{D}$ images is much more difficult than in 2D. Indeed, a hole in a $2 \mathrm{D}$ image is a cavity and thus a region which is a bounded well defined set of points, whereas it is not the case in $3 \mathrm{D}$.

We present in this paper a 3D-hole closing algorithm, which original implementation allows to control the size of the holes that are closed. The closing of the hole is actually performed by building a surface such as a kind of patch which obstructs the hole. As far as we know, this is the first 3D-hole closing algorithm.

\section{Basic notions}

We recall some basic notions of 3D discrete topology (see [8]). 
We denote $E=Z^{3}$. A point $x \in E$ is defined by $\left(x_{1}, x_{2}, x_{3}\right)$ with $x_{i} \in Z$. We consider the three neighborhoods:

$$
\begin{aligned}
& N_{26}(x)=\left\{x^{\prime} \in E ; \operatorname{Max}\left[\left|x_{1}-x_{1}^{\prime}\right|,\left|x_{2}-x_{2}^{\prime}\right|,\left|x_{3}-x_{3}^{\prime}\right|\right] \leq 1\right\} \\
& N_{6}(x)=\left\{x^{\prime} \in E ;\left|x_{1}-x_{1}^{\prime}\right|+\left|x_{2}-x_{2}^{\prime}\right|+\left|x_{3}-x_{3}^{\prime}\right| \leq 1\right\} \\
& N_{18}(x)=\left\{x^{\prime} \in E ;\left|x_{1}-x_{1}^{\prime}\right|+\left|x_{2}-x_{2}^{\prime}\right|+\left|x_{3}-x_{3}^{\prime}\right| \leq 2\right\} \cap N_{26}(x) .
\end{aligned}
$$

We denote $N_{6}^{*}(x)=N_{6}(x) \backslash\{x\}, N_{26}^{*}(x)=N_{26}(x) \backslash\{x\}, N_{18}^{*}(x)=N_{18}(x) \backslash\{x\}$. Two points $x$ and $y$ are said to be $n$-adjacent ( $\mathrm{n}=6,18,26)$ if $y \in N_{n}^{*}(x)$.

We denote $N_{18}^{+}(x)=N_{18}^{*}(x) \backslash N_{6}^{*}(x)$ and $N_{26}^{+}(x)=N_{26}^{*}(x) \backslash N_{18}^{*}(x)$. We call respectively $6,18,26$-neighbors of $x$ the points of $N_{6}^{*}(x), N_{18}^{+}(x)$, and $N_{26}^{+}(x)$. An $n$-path $\pi$ is a sequence of points $x_{0} . . x_{k}$, with $x_{i} n$-adjacent to $x_{i-1}$ for $i=1 . . k$. The length of $\pi$ is equal to $k$. If $x_{0}=x_{k}, \pi$ is closed. The inverse of $\pi$ is the path $\pi^{-1}=x_{k} . . x_{0}$.

An object $X \subset E$ is said to be $n$-connected if for any two points of $X$, there is an $n$-path in $X$ between these two points. The equivalence classes relative to this relation are the $n$-connected components of $X$. The set composed of all the $n$-connected components of $X$ is denoted $\mathcal{C}_{n}(X)$. The set of all $n$-connected components of $X n$-adjacent to a point $x$ is denoted $\mathcal{C}_{n}^{x}(X)$. Note that $\mathcal{C}_{n}(X)$ and $\mathcal{C}_{n}^{x}(X)$ are sets of subsets of $X$ and not sets of points.

As in $2 \mathrm{D}$, if we use an $n$-connectivity for $X$ we have to use another $\bar{n}$-connectivity for $\bar{X}$, i.e. the 6 -connectivity for $X$ is associated to the 18 or the 26 connectivity for $\bar{X}$ (and vice versa). This is necessary for having a correspondence between the topology of $X$ and the topology of $\bar{X}$. Furthermore, it is sometimes necessary to distinguish the 6-connectivity associated with the 18-connectivity and the 6 -connectivity associated with the 26-connectivity. Whenever we will have to make this distinction, a $6^{+}$-notion will indicate a 6 -notion associated with the 18-connectivity. So we can have $(n, \bar{n})=(6,26),(26,6),\left(6^{+}, 18\right)$ or $\left(18,6^{+}\right)$.

If $X$ is finite, the infinite connected component of $\bar{X}$ is called the background, the other connected components of $\bar{X}$ are called the cavities.

\section{Notion of hole}

The notion of a hole is not simple to define. Let $X$ be a subset of $E$. The presence of a hole in $X$ is detected whenever there is a closed path in $X$ that cannot be deformed in $X$ to a single point. For example a hollow ball has one cavity and no hole, a solid torus has no cavity and one hole, a hollow torus has one cavity and two holes. Note that there is a correspondence between the holes of $X$ and the ones of $\bar{X}$, e.g. the complementary of a hollow torus has two holes.

We give below a definition of deformation (see $[1,7]$ ).

Let $p \in X$ be a point, called the base point. Let $\gamma \subset X$ and $\gamma^{\prime} \subset X$ be two closed $n$-paths containing $p$. We say that $\gamma^{\prime}$ is an elementary deformation of $\gamma$, which is denoted $\gamma \sim \gamma^{\prime}$, if $\gamma$ and $\gamma^{\prime}$ are the same but in a little portion $P$ :

- for $n=6, P$ is an unit square (a $2 \times 2$ square);

- for $n=6^{+}, 18,26, P$ is an unit cube (a $2 \times 2 \times 2$ cube).

We say that $\gamma^{\prime}$ is a deformation of $\gamma$ or $\gamma \simeq \gamma^{\prime}$ if there is a sequence of closed $n$-paths $\gamma_{0} . \gamma_{k}$ such that $\gamma=\gamma_{0}, \gamma^{\prime}=\gamma_{k}$ and $\gamma_{i-1} \sim \gamma_{i}$ for $i=1 . . k$. 
The classes of equivalence of the closed paths under the relation $\simeq$ constitute a group $\Pi_{n}(p, X)$ which is the fundamental $n$-group (or Poincaré group) with base point $p$. As in the continuous spaces, $\Pi_{n}(p, X)$ reflects the structure of the holes (or tunnels) in $X$. For example, the fundamental group of a hollow torus is a free group on two generators.

Note that the choice of the base point is free, i.e., $\Pi_{n}(p, X)$ is isomorphic to $\Pi_{n}(q, X)$ provided $p$ and $q$ belong to the same $n$-connected component of $X$.

\section{Topological numbers}

Let $X$ be a subset of $E$. A point $x \in X$ is said to be $n$-simple (for $X$ ) if its removal does not change the topology of the image, i.e., there is a one to one correspondence between the $n$-connected components of $X$, the $\bar{n}$-connected components of $\bar{X}$, the $n$-holes of $X$, and the n-connected components of $X \backslash\{x\}$, the $\bar{n}$-connected components of $\bar{X} \cup\{x\}$, the $n$-holes of $X \backslash\{x\}$, respectively. The set $Y$ is (lower) $n$-homotopic to the set $X$ if $Y$ may be obtained from $X$ by deleting $n$-simple points. If $Y$ is lower $n$-homotopic to $X$, the set $S=X \backslash Y$ is called a (lower) $n$-simple set.

Let $X \subset E$ and $x \in E$. The geodesic n-neighborhood of $x$ inside $X$ of order $k$ is the set $N_{n}^{k}(x, X)$ defined recursively by:

$N_{n}^{1}(x, X)=N_{n b}^{* k}(x) \cap X$ and $N_{n}^{k}(x, X)=\cup\left\{N_{n}(y) \cap N_{26}^{*}(x) \cap X, y \in N_{n}^{k-1}(x, X)\right\}$. In other words $N_{n}^{k}(x, X)$ is the set composed of all points $y$ of $N_{26}^{*}(x) \cap X$ such that there exists an $n$-path $\pi$ from $x$ to $y$ of length less than or equal to $k$, all points of $\pi$, except possibly $x$, belonging to $N_{26}^{*}(x) \cap X$. We give now a definition of topological numbers $[4,3,2]$ :

Definition 1. Let $X$ be a subset of $E$ and $x$ be a point of $E$.

The geodesic neighborhoods $G_{n}(x, X)$ are defined by:

$$
\begin{aligned}
G_{6}(x, X) & =N_{6}^{2}(x, X) ; & G_{6+}(x, X)=N_{6}^{3}(x, X) ; \\
G_{18}(x, X) & =N_{18}^{2}(x, X) ; & G_{26}(x, X)=N_{26}^{1}(x, X) .
\end{aligned}
$$

The topological numbers $T_{n}(x, X)$ are defined by:

$$
\begin{aligned}
T_{6}(x, X)=\# \mathcal{C}_{6}\left[G_{6}(x, X)\right] ; & T_{6+}(x, X)=\# \mathcal{C}_{6}\left[G_{6+}(x, X)\right] \\
T_{18}(x, X)=\# \mathcal{C}_{18}\left[G_{18}(x, X)\right] ; & T_{26}(x, X)=\# \mathcal{C}_{26}\left[G_{26}(x, X)\right] .
\end{aligned}
$$

These numbers lead to a characterization of simple points $([4,3])$ :

Theorem 2. Let $X$ be a subset of $E$ and $x$ be a point of $X$ :

$x$ is an n-simple point $\Leftrightarrow T_{n}(x, X)=1$ and $T_{\bar{n}}(x, \bar{X})=1$.

If we use the $n$-connectivity for $X$ and the $\bar{n}$-connectivity for $\bar{X}, T_{n}(x, X)$ and $T_{\bar{n}}(x, \bar{X})$ are considered for describing the topological characteristics of the point $x$. Other characterizations of simple points have been proposed. The major interest of the characterization based upon the topological numbers is that it is easy to check; furthermore, the topological numbers may be used as general describers of the local topological configuration of a point (see [9]): 
Definition 3. Let $X$ be a subset of $E$ and $x$ be a point of $X$. We denote $T=$ $T_{n}(x, X)$ and $\bar{T}=T_{\bar{n}}(x, \bar{X})$. According to the different possible values of $T$ and $\bar{T}$, the point $x$ is classified as follows:

$T=0:$ isolated point $; \bar{T}=0:$ interior point $; \bar{T} \neq 0:$ border point $T=1, \bar{T}=1$ : simple point

$T=2, \bar{T}=1:$ simple $1 D$ isthmus; $T=1, \bar{T}=2:$ simple $2 D$ isthmus

$T \geq 3, \bar{T}=1$ : simple $1 D$ junction; $T=1, \bar{T} \geq 3$ : simple $2 D$ junction $T \geq 2, \bar{T} \geq 2:$ multiple isthmus

$T \geq 2: 1 D$ isthmus; $\bar{T} \geq 2: 2 D$ isthmus

$T \geq 2$ or $\bar{T} \geq 2 ;$ isthmus

\section{Topological hull}

We introduce the notion of topological hull:

Definition 4. Let $X$ and $Y$ be finite subsets of $E$ such that $X \subset Y$.

We say that $Y$ is a topological hull of $X$, if $Y$ has no holes and no cavities and if, for each $x$ of $Y \backslash X$, the set $Y \backslash\{x\}$ has a hole or a cavity.

The two following theorems will help us to find a way for extracting a topological hull of a set $X$ (the proofs may be found in [3] and in [4]);

Theorem 5. Let $X \subset E$ and $x \in X$. Suppose we remove $x$ from $X$ :

An $n$-component of $X$ is removed $\Leftrightarrow x$ is an isolated point;

An n-component of $X$ is created or an $n$-hole of $X$ is removed $\Leftrightarrow x$ is a $1 D$ isthmus.

Theorem 6. Let $X \subset E$ and $x \in X$. Suppose we remove $x$ from $X$ :

An $\bar{n}$-component of $\bar{X}$ is created $\Leftrightarrow x$ is an interior point;

An $\bar{n}$-component of $\bar{X}$ is removed or an $n$-hole of $X$ is created $\Leftrightarrow x$ is a $2 D$ isthmus.

The following theorem gives a local characterization of the class of sets which are topological hulls relatively to the class of sets which have no holes and no cavities:

Theorem 7. Let $X$ and $Y$ be finite subsets of $E$ such that $X \subset Y$.

Suppose that $Y$ has no cavities and no holes, then:

$Y$ is a topological hull of $X$ if and only if, for each $x$ of $Y \backslash X, x$ is an interior point or a $2 D$ isthmus for $Y$, i.e., $T_{\bar{n}}(x, \bar{Y}) \neq 1$.

Proof. Suppose $Y$ has no cavities and no holes.

i) Suppose $T_{\bar{n}}(x, \bar{Y})=1$ :

- if $T_{n}(x, Y)=1, x$ is simple;

- if $T_{n}(x, Y)=0, x$ is an isolated point and, from Th. 5 and 6 , the only homotopical change induced by the removal of $x$, is the deletion of a component of $Y$; 
- if $T_{n}(x, Y) \geq 2, x$ is a $1 \mathrm{D}$ isthmus and, from Th. 5 and 6 , since $Y$ has no holes, the only homotopical change induced by the removal of $x$, is the creation of a component of $Y$.

In all cases $Y$ cannot be a topological hull.

ii) Suppose $T_{\bar{n}}(x, \bar{Y}) \neq 1$.

- if $T_{\bar{n}}(x, \bar{Y})=0, x$ is an interior point; the removal of $x$ would create a cavity (Th. 6);

- if $T_{\bar{n}}(x, \bar{Y}) \geq 2, x$ is a 2D-isthmus; since Th. 6 and since $Y$ has no cavities, the removal of $x$ would create a hole.

Let $X$ be a subset of $E$. The following definition introduces a binary relation $h$ which is defined on the class of all subsets of $E$ containing $X$.

Definition 8. Let $X$ be a subset of $E$. We define the binary relation $h$ : $\forall U \subset E, \forall V \subset E$ such that $X \subset U$ and $X \subset V$,

$$
V \in h(U) \Leftrightarrow \exists x \in U \backslash X \text {, such that } T_{\bar{n}}(x, \bar{U})=1 \text { and } V=U \backslash\{x\} \text {. }
$$

Let $B \subset E$, with $X \subset B$ and $Y \subset E$. We say that $Y \in h^{\infty}(B)$ if there is a sequence $B=Y^{0}, Y^{1}, \ldots, Y^{k}=Y$, such that $Y^{i} \in h\left(Y^{i-1}\right)$ and such that $h(Y)=\emptyset$.

The following corollary is a direct consequence of the proof of Th. 7 :

Corollary 9. Let $X$ and $B$ be finite subsets of $E$ such that $X \subset B$ and such that $B$ has no cavities and no holes:

for each subset $Y$ of $E$, if $Y \in h^{\infty}(B)$, then $Y$ is a topological hull of $X$.

The Def. 8 and Cor. 9 give a method for extracting a topological hull of a set $X$. We compute a bounding box $B$ which has no cavities and no holes and which contains $X$. We iteratively delete the points of $B$ which do not belong to $X$ and which are not interior point and not $2 \mathrm{D}$ isthmus. We repeat this procedure until idempotence. We have:

Property 10. A topological hull may be obtained in linear time with linear space.

Proof. Let $B$ be a cube which strictly contains $X . B$ has no cavities and no holes. We consider a list $L$ which, at any step of the procedure, contains all border points of the actual set $Y^{i}$. The list $L$ is initialized with border points of $B$. The procedure consists in extracting the first point $x$ of $L$ and checking if this point satisfies the above mentioned conditions for deletion. If yes, the point $x$ is deleted and the points 26 -adjacent to $x$ are inserted in $L$. The procedure is repeated until $L$ is empty.

Note that $L$ necessarily contains all candidates for deletion of the actual set $Y^{i}$. The number of times a given point is inserted in $L$ is bounded by a constant (26).

In figure 1, examples of topological hulls are given. We can see in the example given figure 1 (d) that the added surface may be not centered. In the next section, we will see how to use the distance transform to extract well centered surfaces. 


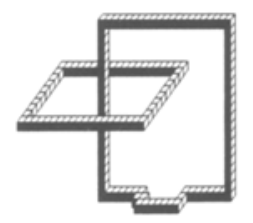

(a)

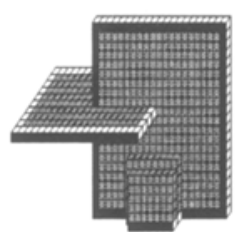

(b)

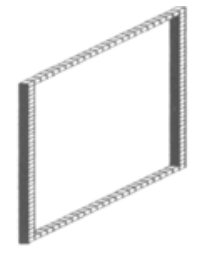

(c)

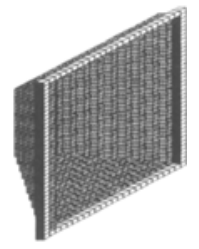

(d)

Fig. 1. (b) and (d) are respectively the topological hulls of (a) and (c).

\section{Topological hull directed by the distance transform}

In the following, we are going to show how the distance transform can be introduced in the computation of the topological hull to control the process to obtain a centered topological hull.

\subsection{Principle}

Let $d$ be a distance over $E=Z^{3}$ and a given $X \subset E$. We call $\psi$ the result of the distance transform such that: $\forall x \in E, \psi(x)=\min _{y \in X}\{d(x, y)\}$

We have seen previously that the topological hull is not unique because several points are topologically speaking equivalent candidates to be used to build such a hull. The most important drawback that we would want to avoid is to obtain a surface which has the tendency to "go away" from the object, as illustrated in the figure 1 (d).

We use the distance transform as a constraint in order to restrict the choice of the possible points to build the topological hull. The points are sorted according to their decreasing distance from the object. Then, the previously described algorithm to construct the topological hull is applied, but we process the points according to their decreasing distance order. The furthest points from the object are going to be processed first, and then, successively, the closest points. When a point is processed, it is eliminated if it does not satisfy the surviving criteria. In this case, its neighborhood is scanned and the points which are not already inserted in the list to be processed are then inserted. By ordering the points according to their distance from the object and processing them in the decreasing order, we are going to eliminate first the furthest points, and thus, the first points which are going to survive to the elimination process will be the closest to the object.

In discrete spaces, one can find several definitions of distance $[5,6]$. For example we will call $D_{6}$ the distance in which all the points in $N_{6}^{*}(x)$ are considered distant of 1 from the point $x$, and we can define similarly $D_{26}$ in which the points in $N_{26}^{*}(x)$ are at distance 1 from $x$. The choice of the distance has an influence on the resulting topological hull. The figure 2 presents an elementary object (a) 
which has been processed by different types of classical discrete distances. One can note that the shape of the produced surface differs.

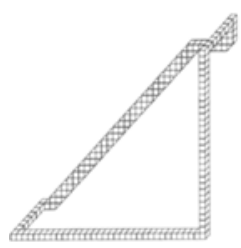

(a)

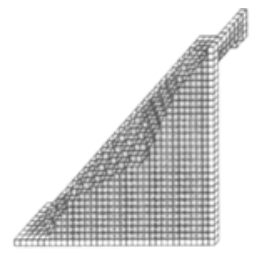

(b)

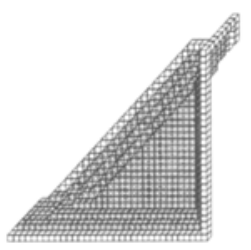

(c)

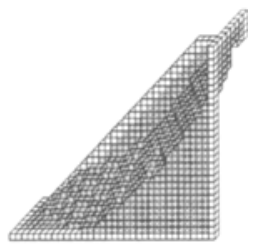

(d)

Fig. 2. (a): Initial object; The topological hulls using : (b)the $D_{6}$ distance, (c): the $D_{26}$ distance, (d): The chamfer distance (3-4-5).

\subsection{Properties}

The topological hull directed by the distance transform has several interesting properties which will be the topic of a future article. We just wish to mention the two main ones here.

The first interesting property of the surfaces built by the topological hull directed by the distance transform is that it looks centered. By centered, we mean that the points which are actually chosen to build the surface from all the possible ones belong to the set of the closest points from the object, having the effect of building a surface close to the object. The consequence is to avoid to obtain such a topological hull as previously illustrated in figure 1 (d).

The second important property is that we have the ability to control the size of the holes which are going to be closed. Indeed, as we previously mentioned, the generic holes closing process is going to process all of the points which have been sorted according to the decreasing order of their distance value. In other words, the process starts with the highest distance value found in the image, down to the lowest. Let us suppose now that we do not start from the highest distance value, but from an arbitrary given value $\epsilon$. Then, all the points with a greater distance value will not be modified, and thus the holes which size is greater than $\epsilon$ will not be closed. We will call $\epsilon$ the threshold. The notion of size that we are mentioning directly comes from the distance transform. The size of a hole can be seen as the smallest radius of the spheres (in the sense of the chosen distance) which one can fit in the hole.

The figure 3 presents an example of the control of the size of the closed holes. The initial object (a) is a half-sphere with several holes of various sizes. In figure 3 (b) we set a hole size of $\epsilon=2$, and one can see that the smallest holes have been closed. Then on (c) we set the threshold $\varepsilon$ to the maximum size 


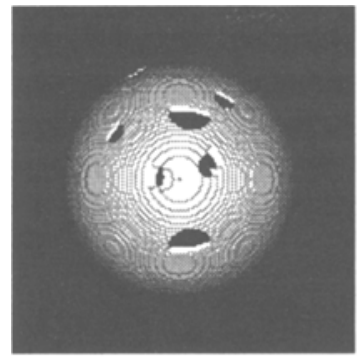

(a)

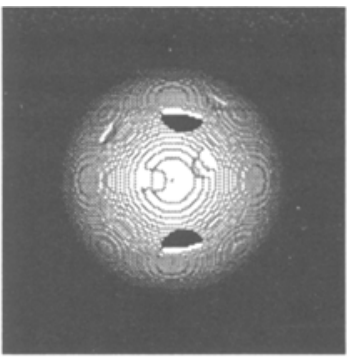

(b)

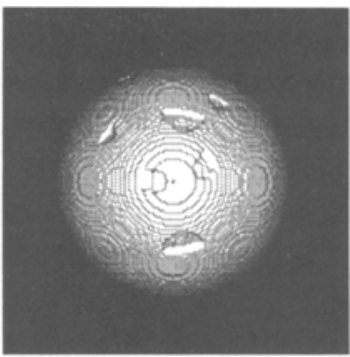

(c)

Fig. 3. (a): initial image of a half-sphere with holes. (b): Result of the algorithm with $\epsilon=2$. (c): Result of the algorithm with $\epsilon>2$

it can be seen that all the holes have been closed by the construction of the corresponding patch of surfaces.

Another example is presented on figure 4 on a biomedical image of a vertebra. The figure 4 (a), (b) and (c) illustrate how the threshold can be used to keep relevant holes and eliminate the holes which correspond to noise. (a) is the initial image resulting from the segmentation process applied to the acquisition. (b) is the result with $\epsilon=2$ and several irrelevant holes remaining in the main body of the vertebra may be observed. (c) is obtained with a better suited value of the threshold $\epsilon=3$.

The influence of the discrete distance can be seen on the same figure 4 . The figure 4 (d), (e) and (f) present the centered topological hull obtained with a maximum value of the threshold and respectively the discrete distances $D_{6}, D_{26}$ and the chamfer(3-4-5) distance.

\section{Complexity of the centered topological hull construction}

Let us consider that we have an image with $n$ points. We can claim that in order to build the topological hull, we need to access each point of the image at least once leading to a complexity of at least $O(n)$. In addition to that, as we have to process the points in the decreasing order of their distance value, we must sort them according to their distance value, and finally, perform the individual deletion process of each point.

Concerning the sorting step, we must distinguish two cases according to the information we have about the possible values of the distance transform.

Suppose that we do not have any information about the possible values of the distance transform other that $\psi$ satisfies the definition of the distance and that the distance values are integers. Then, while we scan the initial image, we have to sort the points one by one according to their distance value when we 


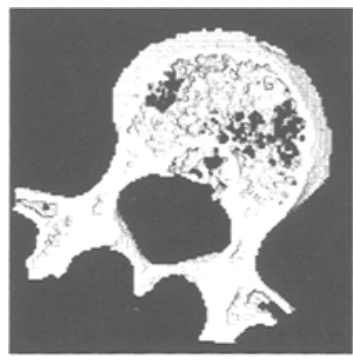

(a)

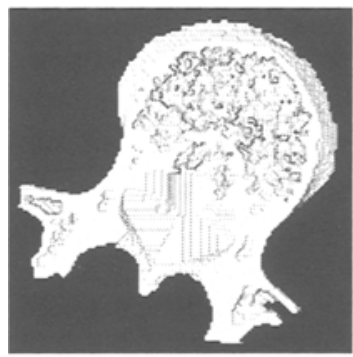

(d)

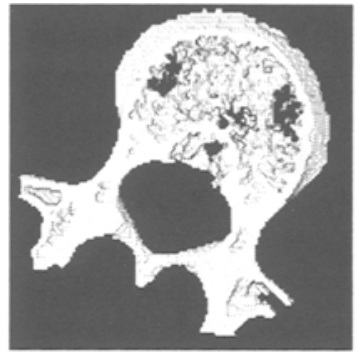

(b)

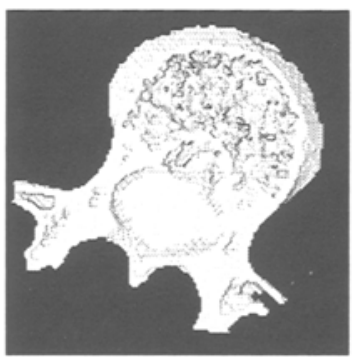

(e)

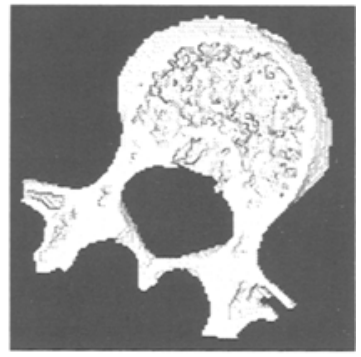

(c)

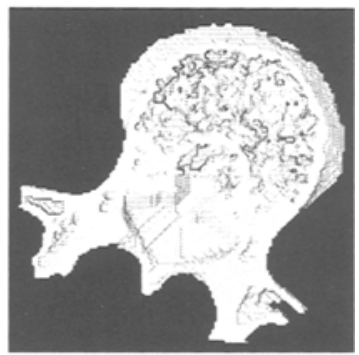

(f)

Fig. 4. Application of the $3 \mathrm{D}$ holes closing algorithm to an image of a vertebra. The 26-connectivity for the object is used.(a) : initial image (7 slices). (b) : $\epsilon=2$. (c) : $\epsilon=3$. (d), (e), (f) : Maximum value of $\epsilon$ using respectively the $D_{6}, D_{26}$ and the chamfer(3-4-5) distances.

encounter them. This step can be bounded by $O(n \times \log (n))$ leading to a global complexity of $O(n \times \log (n))$.

Suppose that we know that $\psi$ will produce the $k$ first integer values, then the sorting may be done in an array of size $k$. When we encounter a value of distance transform, it is then possible to access in constant time its corresponding list of points by an index and then to add still in constant time the point in the list. Thus, in this later case, we have a linear complexity of $O(n)$.

We do not include in our complexity evaluation the computation of the distance transform which may be dependant of the discrete distance which is used. Furthermore, in a more general approach a function different from a distance transform might be used to sort the points, therefore leading to a different complexity.

\section{Algorithm : hierarchical list}

As seen in the previous section, during the conditional shrinking process, we start by suppressing the points which are the furthest from the feature elements. This method is implemented by using a hierarchical list. 
Description of the hierarchical list. A hierarchical list is an ordered group of FIFO lists. The lists are ordered among themselves according to a certain criteria. We will call this criteria a priority level because it refers to the stage when a given list is going to be processed. In the case of our algorithm, the elements of the lists will be the points to be considered in the shrinking operation and the priority level of the list will be the distance value of the points in the list. At the initial step, all the points with the same distance transform value belong to the same list.

During the extraction process, the element processed is the oldest one in the highest priority list, whereas during the storage process, the elements are inserted in the list corresponding to their priority level. When all the elements are extracted, this list becomes empty and the elements of the list with the immediate inferior priority are processed.

It can happen that an element having a priority higher than the current one has to be inserted. In this case, the element is inserted in its corresponding list and this list becomes the current one since its priority level is the highest one now.

The algorithm. The entries of the algorithm are a distance transform image $\psi$ and a distance threshold $\epsilon$, and the result is the centered topological hull $H$ of the initial image object in which the holes of size equal or less than $\epsilon$ are closed. The $n$-connectivity is used for the object and the $\bar{n}$-connectivity for the complementary. In addition to that, we will use a binary image $E$ which associates a boolean flag (TRUE or FALSE) to each point of the image to let us know if the corresponding point already belongs to the hierarchical list. We also use a function $\operatorname{FIRST}(L)$ to extract the first element of a list $L$.

The algorithm is represented on figure 5 .

During the initialization step, we create a hierarchical list $L$ filled with the points of the image border, i.e. the points of the image that have at least one neighbor in the bounding box $B$ (see Section 5). Each point $x$ is inserted in the FIFO list $L[\psi(x)]$ with a priority level corresponding to its distance value $\psi(x)$, then the flag $E[x]$ is set to indicate the insertion of the point $x$ in the hierarchical list.

The algorithm successively processes the lists in their decreasing priority order starting from $N$. The priority 0 corresponds to the object points which are excluded from the shrinking process.

For the current priority list, we extract the first point $x$ and the flag $E[x]$ is reset. Then this point is suppressed from the topological hull $H$ if its distance value is higher than $\epsilon$ or if it is not a surface point, i.e. if its corresponding topological number $\bar{T}$ is equal to 1 . When the point $x$ is suppressed, the points of the 26-neighborhood of $x$ which are not already in the list are inserted in the hierarchical list. As mentioned before, these points are inserted in the list corresponding to their distance value. If there is a point in a higher priority list, 


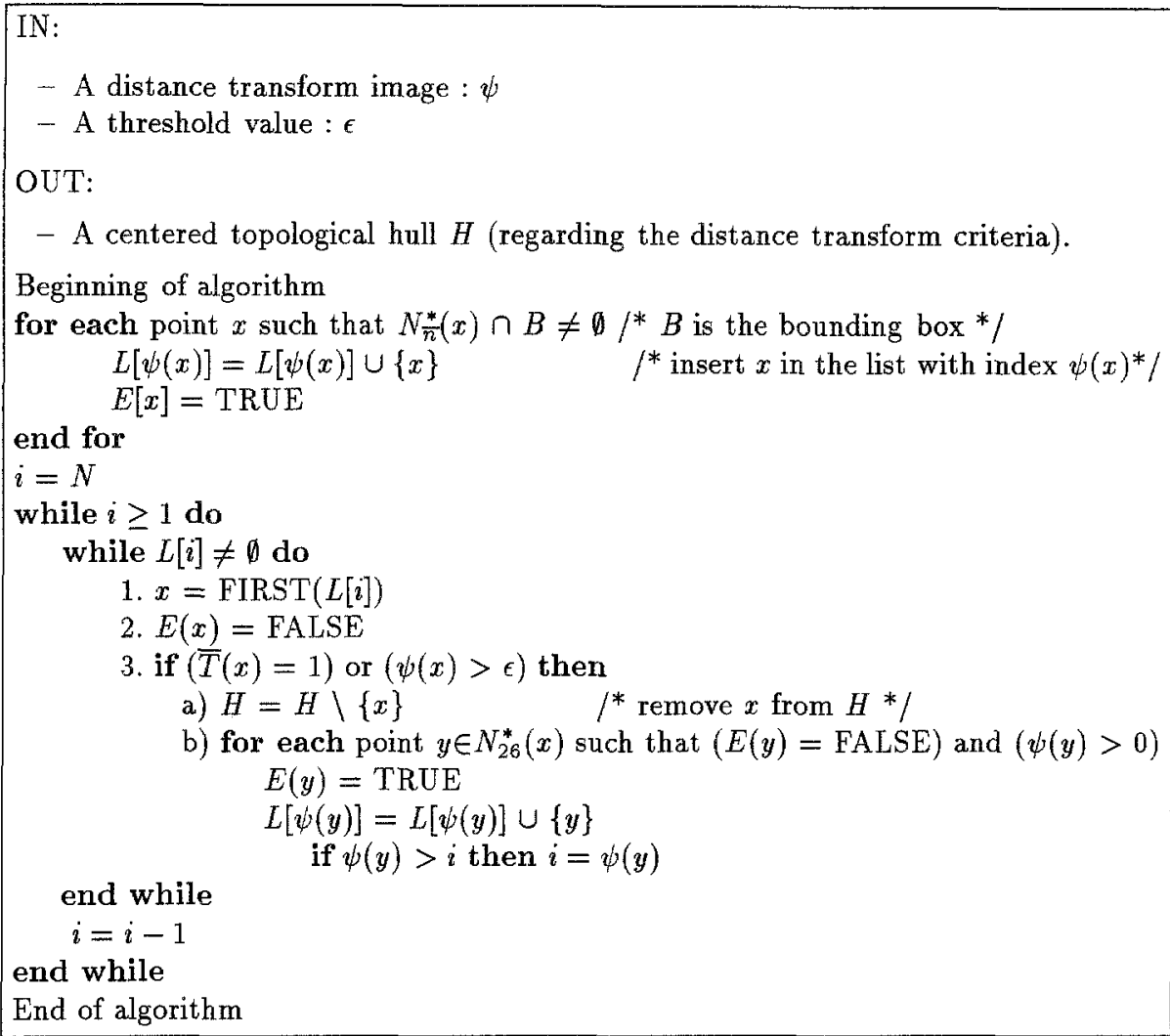

Fig. 5. The 3D-hole closing algorithm

this list becomes the current one.

The process ends when all the FIFO lists become empty and then the remaining points are the ones of the object in addition with the ones which close the holes of size $\epsilon$ and less.

\section{Conclusion}

A 3D-hole closing algorithm has been presented. It is based on some properties of the topological numbers and on the principle of the topological hull. It has the originality to close the $3 \mathrm{D}$-holes of different sizes in order to preserve those which are not actually resulting from a noise effect. In addition to that, our algorithm has the advantage to be linear in time complexity.

The method that we have proposed fits into the topological image processing domain. This domain has a large range of applications, such as the biomedical area (see $[10,11])$. 


\section{References}

1. G. Bertrand. Characterization of three-dimensional holes. Internal report, 1992.

2. G. Bertrand. On the computation of topological numbers. In 4 th Discrete geometry for computer imagery (DGCI'94), pages 137-146, September 1994.

3. G. Bertrand. Simple points, topological numbers and geodesic neighborhoods in cubic grids. Pattern Recognition Letter, 15:1003-1011, 1994.

4. G. Bertrand and G. Malandain. A new characterization of 3d simple points. Pattern Recognition Letter, 15:169-175, 1994.

5. G. Borgefors. Distance transforms in arbitrary dimension. Computer Vision, Graphics and Image Processing, 27:321-345, 1984.

6. J.M. Chassery and A. Montanvert. Géométrie discrète en analyse d'images. Hermès, 1991.

7. T.Y Kong. A digital fundamental group. Computer Graphics, 13:159-166, 1989.

8. T.Y. Kong and A. Rosenfeld. Digital topology: introduction and survey. Computer Vision, Graphics and Image Processing, 48:357-393, 1989.

9. G. Malandain, G. Bertrand, and N. Ayache. Topological segmentation of discrete surfaces. Int. Journal of Comp. Vision, 10(2):183-197, 1993.

10. G. Malandain and S. Fernández Vidal. Topologically correct skeleton in $n-d$. In 5th Discrete geometry for computer imagery (DGCI'95), pages 199-208, September 1995.

11. J.F. Mangin, V. Frouin, I. Bloch, J. Regis, and J. Lòpez-Krahe. From 3d magnetic resonance images to structural representations of the cortex topography using topology preserving deformations. Journal of Mathematical Imaging and Vision, 5:297-318, 1995. 